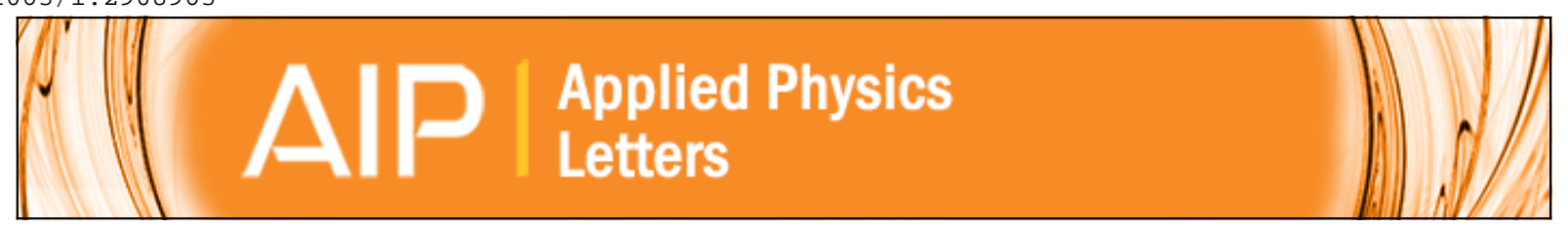

\title{
A piezoelectric single crystal traveling wave step motor for low-temperature application
}

Shuxiang Dong, Li Yan, D. Viehland, Xiaoning Jiang, and Wesley S. Hackenberger

Citation: Applied Physics Letters 92, 153504 (2008); doi: 10.1063/1.2908963

View online: http://dx.doi.org/10.1063/1.2908963

View Table of Contents: http://scitation.aip.org/content/aip/journal/apl/92/15?ver=pdfcov

Published by the AIP Publishing

\section{Over 700 papers \&}

presentations on

multiphysics simulation visw now

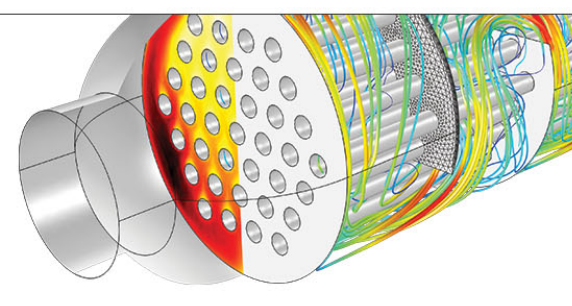




\title{
A piezoelectric single crystal traveling wave step motor for low-temperature application
}

\author{
Shuxiang Dong, ${ }^{1,2, a)}$ Li Yan, ${ }^{2}$ and D. Viehland ${ }^{2}$ \\ ${ }^{1}$ Advanced Materials \& Nanotechnology, College of Engineering, Peking University, Beijing 100871, China \\ ${ }^{2}$ Materials Science and Engineering, Virginia Tech, Blacksburg, Virginia 24061, USA \\ Xiaoning Jiang ${ }^{3}$ and Wesley S. Hackenberger ${ }^{3}$ \\ ${ }^{3}$ TRS Technologies, Inc., State College, Pennsylvania 16801, USA \\ (Received 18 December 2007; accepted 25 March 2008; published online 15 April 2008)

\begin{abstract}
A piezoelectric $\mathrm{Pb}\left(\mathrm{Mg}_{1 / 3} \mathrm{Nb}_{2 / 3}\right) \mathrm{O}_{3}-\mathrm{PbTiO}_{3}$ single crystal traveling wave rotary motor operated in a stepping motion for cryogenic actuations was developed and characterized. This single crystal stepping motor had a torque of $\geqslant 1.5 \mathrm{~kg} \mathrm{~cm}$, an excess of 1800 steps per revolution about its axis, and a power consumption of $\leqq 2 \mathrm{~W}$ by using a step motion with $25 \%$ duty cycle. We then show that this single crystal motor can successfully operate at $77 \mathrm{~K}$ (liquid nitrogen) under a load. (c) 2008 American Institute of Physics. [DOI: 10.1063/1.2908963]
\end{abstract}

Cryogenic motors with a large torque, high stepping resolution, and low power consumption are desired for cryogenic actuation applications, including cryogenic passive optics; adaptive structure drives for shape, position, and force controls in spaceborne and airborne platforms; tunable cryogenic microwave devices; and other cryogenic scientific instruments. ${ }^{1-5}$ Current generations of piezoelectric $\mathrm{Pb}(\mathrm{Zr}, \mathrm{Ti}) \mathrm{O}_{3}$ (PZT) based ultrasonic step motors/actuators have high thrust to weight ratios, have high stepping or position resolutions, have no parasitic magnetic fields, and have frictional locking in the power-off state ${ }^{6-10}$ but have limitations for operation at cryogenic temperatures. Giant magnetostrictive actuators with superconducting coils work well at low temperatures of $<77 \mathrm{~K}$ but are difficult to drive and do not have as much displacement as at room temperature. ${ }^{11}$ Piezoelectric single crystals, such as $\mathrm{Pb}\left(\mathrm{Mg}_{1 / 3} \mathrm{Nb}_{2 / 3}\right) \mathrm{O}_{3}-\mathrm{PbTiO}_{3} \quad$ (PMN-PT) or $\mathrm{Pb}\left(\mathrm{Zn}_{1 / 3} \mathrm{Nb}_{2 / 3}\right) \mathrm{O}_{3}-\mathrm{PbTiO}_{3}$, exhibit three to five times the strain of conventional piezoelectric PZT ceramics at room temperature. Even at an extremely low temperature of $30 \mathrm{~K}$, the piezoelectric performances (piezoelectric constants $d_{33}$ and $d_{31}$ ) of the single crystals are still high as compared to room-temperature PZT ceramics. ${ }^{12}$ Accordingly, they are good candidates for cryogenic ultrasonic motor driving elements. Recently, some piezoelectric single crystal based motors have been developed. ${ }^{13,14}$ In a prior report, ${ }^{14}$ we developed a PMN-PT single crystal linear-type motor, which had a large driving force of $200 \mathrm{gf}$ and a high resolution of $20 \mathrm{~nm}$, which was also successfully operated at liquid-nitrogen temperatures $(77 \mathrm{~K})$. Here, we will report on a single crystal rotary-type step motor that has a large torque output and a high step resolution with a low profile and light weight, aiming at cryogenic applications.

Our piezoelectric single crystal motor was made of nine PMN-PT single crystal isosceles trapezoids, which were bonded on a titanium-alloy ring and operated on a nine wavelengths $(9 \lambda)$ flexure traveling wave principle. ${ }^{15} \mathrm{We}$ used a Ti-alloy stator element because of its high strength, low weight, and consistent performance at lower tempera- tures. Each PMN-PT trapezoid was poled in the thickness direction and operated in a $d_{31}$ mode. The assembled PMN$\mathrm{PT} / \mathrm{Ti}$-alloy composite ring was a $9 \lambda$ unimorph configuration that formed a $9 \lambda$ bending mode, and therefore, a $9 \lambda$ traveling wave could be excited under one pair of sine wave and cosine wave signals (i.e., a codrive). This traveling wave drove a rotor to which it was contacted via frictional forces. Figures 1(a) and 1(b) illustrate the configurations and the measured impedance spectrum of the PMN-PT/Ti-alloy traveling wave motor (using a Precision impedance analyzer, Agilent 4294A), respectively. The mass of the assembled single crystal motor was less than $200 \mathrm{~g}$.

By considering one $\lambda / 2$ unimorph from the PMN-PT/Tialloy composite ring, the first bending mode resonance frequency (i.e., $9 \lambda$ bending mode frequency) can be approximated as

$$
f_{0}=\frac{\pi d \beta_{1}^{2}}{4 \sqrt{3} l} \bar{\nu},
$$

where $\beta_{1}$ is a constant, $d$ and $l$ are the total thickness and mean length of the $\lambda / 2$ arc segment in the stator ring, and $\bar{v}$ is the mean acoustic velocity of the composite segment, which is given by ${ }^{16}$

$$
\bar{\nu}^{2}=\left(n_{m} Y_{m}+n_{p} Y_{p}\right) / \bar{\rho}, \quad \bar{\rho}=\left(\rho_{p} h_{p}+\rho_{m} h_{m}\right) /\left(h_{p}+h_{m}\right),
$$

where $n_{m}$ is the thickness ratio of the metal plate to the composite one, $n_{p}$ is the thickness ratio for the piezoelectric plate, $h_{m}$ and $h_{p}$ are the thicknesses of the metal and piezoelectric plates, respectively, and $\rho_{m}, \rho_{p}$, and $\bar{\rho}$ are the mass densities for the metal, piezoelectric, and composite plates, respectively. Please note that there is an unstrained face (i.e., neutral face) in the composite ring stator, where the stator can be held with a minimum traveling wave energy loss. The following relationship gives the neutral face position of the composite ring stator:

$$
\begin{gathered}
t_{n}=\frac{Y_{2} h_{m}^{2}+Y_{1} h_{p}^{2}+2 Y_{1} h_{m} h_{p}}{2\left(Y_{2} h_{m}+Y_{1} h_{p}\right)}, \quad Y_{1}=\frac{Y_{p}}{1-\nu_{p}^{2}}, \\
Y_{2}=\frac{Y_{m}}{1-\nu_{m}^{2}} .
\end{gathered}
$$



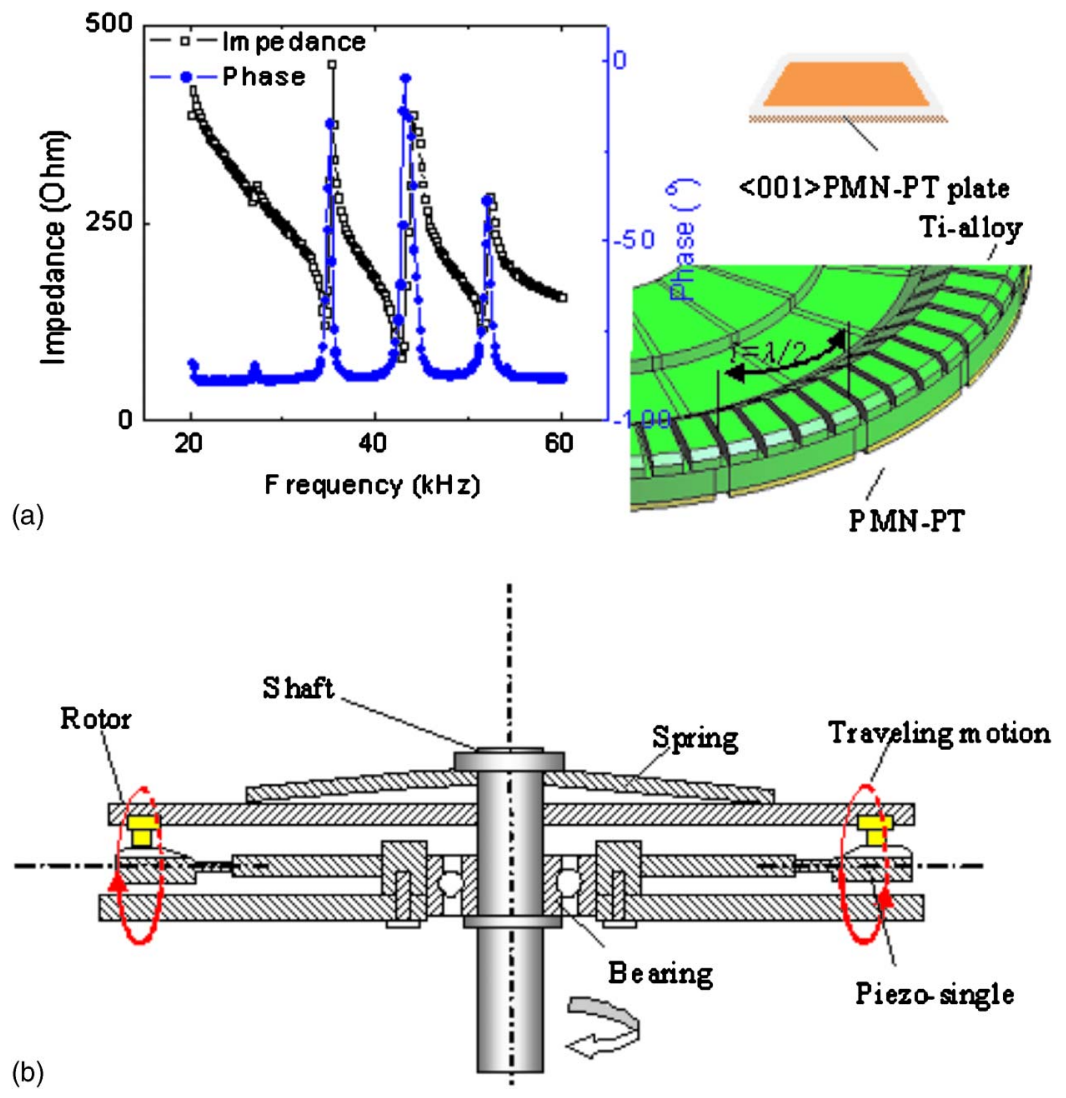

FIG. 1. (Color online) PMN-PT single crystal/Ti-alloy $9 \lambda$ traveling wave motor: (a) PMN-PT/Ti-alloy stator and its impedance spectrum and (b) motor configuration.
By following conditions (1)-(3), using material parameters $\left(Y_{p}=12 \times 10^{9} \mathrm{~Pa}, v_{p}=0.3, \rho_{p}=8040 \mathrm{~kg} / \mathrm{m}^{3}, Y_{m}=108\right.$ $\left.\times 10^{9} \mathrm{~Pa}, v_{m}=0.361, \rho_{p}=4500 \mathrm{~kg} / \mathrm{m}^{3}\right)$, supposing that the outer and inner diameters of the composite stator ring are $D_{\text {out }}=60 \mathrm{~mm}$ and $D_{\text {in }}=45 \mathrm{~mm}$, assuming a resonance frequency for the stator of $40 \mathrm{kHz}$, assuming that the thickness of the PMN-PT trapezoid is $0.5 \mathrm{~mm}$ and that the mean length of one $\lambda / 2$ unimorph is $l=\pi \bar{R} / 9$ [where $\bar{R}=\left(D_{\text {out }}+D_{\text {in }}\right) / 4$ ], and neglecting teeth effects, we can find that the Ti-alloy ring's thickness is $2.03 \mathrm{~mm}$ and that the neutral face is near the middle face of the Ti-alloy ring at $1.02 \mathrm{~mm}$.

First, we analyzed the impedance spectrum of the assembled PMN-PT/Ti-alloy composite stator, as shown in Fig. 1(a). Three resonance peaks were observed: the middle peak at $f=44 \mathrm{kHz}$ corresponds to the ninth order bending mode of the ring stator, whereas the first and third peaks correspond to the lower- and higher-order bending modes. Compared to a PZT-ceramic/brass-alloy ring stator (Fukoku Shinsei ultrasonic motor, USR60, Japan), the PMN-PT/Ti-alloy stator had a four to five times wider resonance peak with a three times lower impedance due to its relatively lower mechanical quality factor $Q_{m}$. From this spectrum, we can expect that our PMN-PT motor can work at lower working voltages over a wider frequency range compared to a PZT-ceramic/brass ring stator. This feature is important for practical applications, in particular, when working under cryogenic circumstances.

Next, we measured our single crystal motor's torque speed and power output performances after the composite stator was installed on the base of the motor, as illustrated in Fig. 1. Figure 2(a) shows the torque-speed relationship, which was measured by a weight method. The maximum torque output under a $100 \mathrm{Vpp}$ drive was about $1.5 \mathrm{~kg} \mathrm{~cm}$, whereas the maximum rotary speed without a load under the same drive voltage was $60 \mathrm{rpm}$. The corresponding maxi- mum power output and efficiency of the motor are $0.45 \mathrm{~W}$ and $6 \%$ (from the motor itself) at a torque $\tau=1 \mathrm{~kg} \mathrm{~cm}$, respectively. Note that the efficiency of the PZT-ceramic/brassalloy ring-type motor under the same operation condition is also low $(\sim 8 \%)$. The efficiency is strongly related to the interface and contact pressures between the stator and rotor of a piezoelectric motor due to a frictional driving mechanism. ${ }^{6,7}$ The higher efficiency up to $40 \%$ might be achieved under a smaller contact pressure and perfect interface, but it usually results in a lower torque but a much higher speed output. As a step motor, the torque, step angular resolution, and response speed are more important issues. We then measured the step motion and response time of the PMN-PT motor with an optical-fiber sensor (MTI2000 Fotonic Sensor) and a digital oscilloscope. Figure 2(b) shows the detected stepping displacement of the PMN-PT motor on driving a stage via a screw. By adjusting the impulse width of a control signal, the stepping angle of the PMN-PT motor was precisely controlled. For an impulse width of $1.2 \mathrm{~ms}$, the minimum step displacement was $\sim 0.1 \mu \mathrm{m}$, which corresponds to a minimum rotational angle of $0.072^{\circ}$ step. These results clearly show that our PMN-PT motor has an extremely high step angle resolution. Figure 2(c) shows the PMN-PT motor's displacement response on applying a pair of drive signals. Again, a very fast response time of $<3.6 \mathrm{~ms}$ was observed. In addition, we measured the power consumption operated in a step motion using a $25 \%$ duty cycle: it was as low as $2 \mathrm{~W}$. However, when the same motor was operated in a continual state, the power consumption was $\sim 8 \mathrm{~W}$. By considering that actuators mostly operate in a step motion, these results demonstrate that our PMN-PT motors have a potential for low power consumption.

Finally, we measured the PMN-PT motor's cryogenic performances. We fixed the PMN-PT motor on a base that 


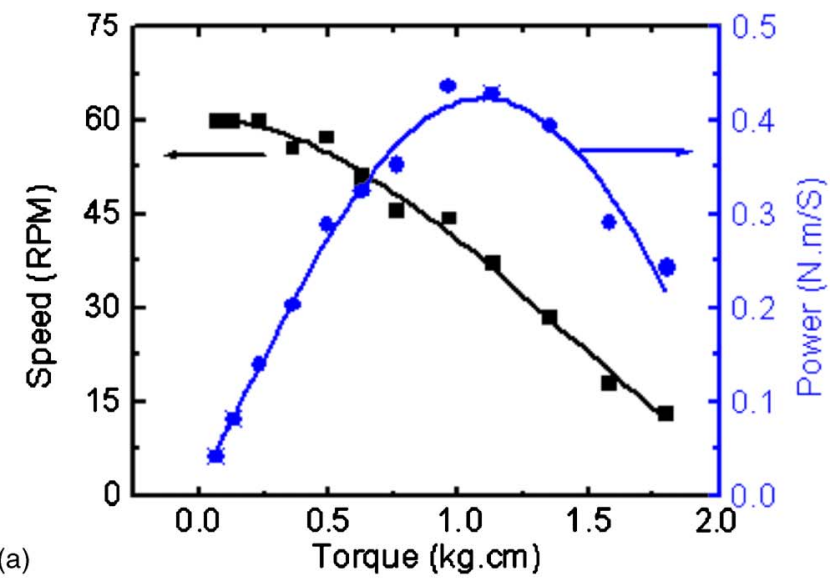

(a)
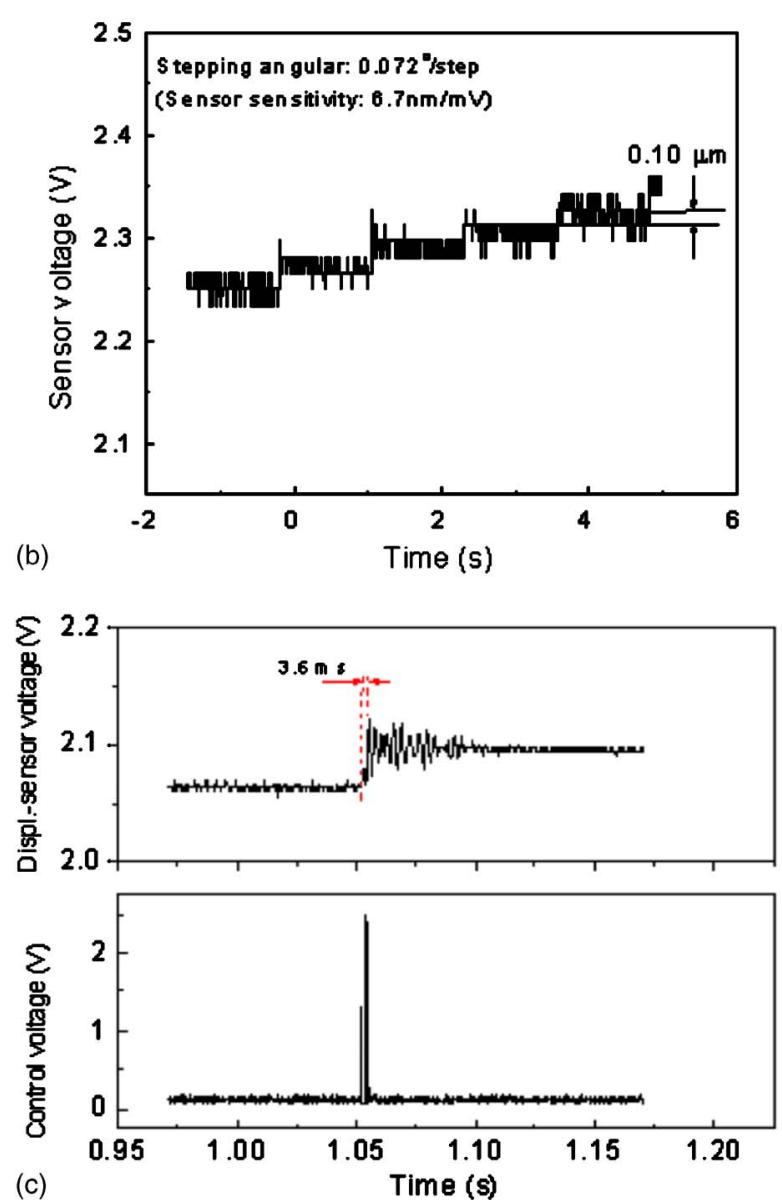

FIG. 2. (Color online) PMN-PT/Ti-alloy traveling wave motor performances at room temperature: (a) measured speed-torque and power output relationship, (b) step responses, and (c) time response.

was placed in a cryogenic chamber. A thermal couple was placed at the bottom of the motor in order to monitor its temperature. A scale disk was placed on top of the motor and a needle was fixed on the motor's output axis, which could be used to indicate the motor's motional direction. The measurement system was then cooled by liquid nitrogen to $\leqq-200{ }^{\circ} \mathrm{C}$. The results demonstrated that our PMN-PT motor worked well at liquid-nitrogen temperatures even when a load of $300 \mathrm{~g}$ was placed on its output axis. We believe that these results demonstrate the potential of our PMN-PT/Ti alloy as a precise and low-profile cryogenic actuator. This device could have important applications in cryogenic microscopy and micropositioning, deployable space structures, and optic platforms.

In summary, a piezoelectric single crystal PMN-PT/Tialloy traveling wave ultrasonic motor has been developed. The maximum torque output was $>1.5 \mathrm{~kg} \mathrm{~cm}$, the minimum step resolution was $<0.072 \%$ step, and the power consumption at a $25 \%$ duty cycle was $\sim 2 \mathrm{~W}$. This motor successfully operated in a liquid-nitrogen environment of $77 \mathrm{~K}$ under a load. The findings demonstrate that PMN-PT single crystal motors have a significant torque improvement, relative to PZT ones, under relatively low voltages.

This project is sponsored by NASA under Grant No. NNL07AA94P.

${ }^{1}$ T. C. van den Dool, F. Kamphues, W. L. M. Gielesen, and B. C. Braam, Proc. SPIE 6692, 66920A-1 (2007).

${ }^{2}$ M. L. Mulvihill, R. J. Shawgo, R. B. Bagwell, and M. A. Ealey, J. Electroceram. 8, 121 (2002).

${ }^{3}$ R. Nalbandian, Proceedings of the Tenth European Space Mechanisms and Tribology Symposium, San Sebastián, Spain, 2003 (unpublished), p. 217.

${ }^{4}$ G. Behme, A. Richter, M. Suptitz, and Ch. Lienau, Rev. Sci. Instrum. 68, 3458 (1997).

${ }^{5}$ A. Garnier, T. Bourouina, H. Fujita, T. Hiramoto, E. Orsier, and J.-C. Peuzin, Sens. Actuators, A 84, 156 (2000).

${ }^{6} \mathrm{~K}$. Uchino, Piezoelectric Actuators and Ultrasonic Motors (Kluwer Academic, Boston, 1997).

${ }^{7}$ T. Sashida and T. Kenjo, An Introduction to Ultrasonic Motors (Clarendon, Oxford, 1993).

${ }^{8}$ S. X. Dong, S. P. Lim, K. H. Lee, and K. Uchino, IEEE Trans. Ultrason. Ferroelectr. Freq. Control 50, 361 (2003).

${ }^{9}$ S. X. Dong, H. W. Kim, M. T. Strauss, K. Uchino, and D. Viehland, IEEE Trans. Ultrason. Ferroelectr. Freq. Control 51, 1240 (2004).

${ }^{10}$ S. X. Dong, S. Wang, W. Shen, and L. Li, IEEE/ASME Trans. Mechatron. 5, 325 (2000).

${ }^{11}$ C. H. Joshi, Proc. SPIE 4131, 240 (2000).

${ }^{12}$ X. Jiang, P. W. Rehrig, W. S. Hackenberger, E. Smith, S. X. Dong D. Viehland, J. Moore, and B. Patrick, Proc. SPIE 5761, 253 (2005).

${ }^{13}$ L. Luo, H. Zhu, C. Zhao, H. Wang, and H. Luo, Appl. Phys. Lett. 90, 052904 (2007).

${ }^{14}$ S. X. Dong, L. Yan, N. Wang, D. Viehland, X. Jiang, P. Rehrig, and W. Hackenberger, Appl. Phys. Lett. 86, 053501 (2005).

${ }^{15}$ T. Sashida, Mechanical Automation 15, 31 (1983) [in Japanese].

${ }^{16} \mathrm{~S}$. X. Dong, K. Uchino, L. Li, and D. Viehland, IEEE Trans. Ultrason. Ferroelectr. Freq. Control 54, 1240 (2007). 